\title{
THE EXISTENCE OF WAVE OPERATORS IN SCATTERING THEORY
}

\author{
BY MARTIN SCHECHTER
}

Communicated by P. Halmos, December 9, 1976

In 1957, Cook [1] gave a sufficient condition for the existence of wave operators. Up to the present time, results obtained by his method are the best, if one is interested merely in proving the existence of the wave operators, and oscillations are not taken into account (cf. [2], [3], [4]). The purpose of this paper is to present a generalization of Cook's criterion which gives stronger results in applications. We exhibit some of these here. Our criterion comes from a new theory of scattering which is much deeper than that required for Cook's theorem.

1. The abstract theory. Let $H_{0}, H$ be selfadjoint operators on a Hilbert space $\mathrm{H}$, with resolvents $R_{0}(z), R(z)$, respectively. Define

$$
j(z, f, g)=\frac{z-\bar{z}}{2 \pi i}\left(R_{0}(z) f,\left[R(z)-R_{0}(z)\right] g\right),
$$

and, when it exists,

$$
J_{I}^{ \pm}(f, g)=\lim _{0<a \rightarrow 0} \int_{I} j(s \pm i a, f, g) d s,
$$

where $I$ is an interval. If $I=(a, b)$, we put $\nu_{I}=\min (-a, b)$.

THEOREM 1. Suppose $f$ is in the subspace of continuity of $H_{0}$ and that for each bounded interval I with $\nu_{I}$ sufficiently large there is a dense set $S_{I} \subset H$ such that $J^{+}(f, g)$ exists for each $g \in S_{I}$. Assume also that

$$
\limsup _{t \rightarrow+\infty} J_{I}^{+}\left(e^{-i t H_{0}} f, e^{-i t H_{0}} f\right) \rightarrow 0 \quad \text { as } v_{I} \rightarrow \infty
$$

for each such I. Then the limit

$$
W_{+} f=\lim _{t \rightarrow \infty} e^{i t H} e^{-i t H} 0 f
$$

exists. If the assumptions hold with the plus signs replaced by minus signs, then the limit (4) exists as $t \rightarrow-\infty$.

2. The generalization of Cook's theorem. Again let $H_{0}, H$ be selfadjoint operators on a Hilbert space $H$. Suppose there are a Hilbert space $K$ and oper-

AMS (MOS) subject classifications (1970). Primary 47A40; Secondary 35J10, 35P25, $81 \mathrm{~A} 45$. 
ators $A, B$ from $H$ to $K$ such that $A$ is $H_{0}$-bounded, $B$ is $H$-bounded and

$$
(H u, v)=\left(u, H_{0} v\right)+(B u, A v)_{K}, \quad u \in D(H), v \in D\left(H_{0}\right) .
$$

The following is a consequence of Theorem 1.

THEOREM 2. If $e^{-i t H_{0}} f \in D(A)$ for $t>a$ and

$$
\int_{a}^{\infty}\left\|A e^{-i t H_{0}} f\right\| d t<\infty
$$

then the limit (4) exists.

3. Applications. The following are consequences of Theorem 2. Let $H_{0}$ be the selfadjoint operator in $L^{2}\left(E^{n}\right)$ corresponding to $-\Delta$. If there are constants $m, \gamma>1$ such that

$$
\|\nabla u\|^{2}+m^{2}\|u\|^{2}+\gamma(V u, u) \geqslant 0, \quad u \in C_{0}^{\infty},
$$

then $H_{0}+V$ has a selfadjoint forms extension $H$ (cf. [9]).

Theorem 3. Assume

$$
(|V| u, u) \leqslant C\left(\|\nabla u\|^{2}+\|u\|^{2}\right), \quad u \in C_{0}^{\infty},
$$

and that for each $y \in E^{n}$ there is an $a>0$ such that

$$
\int_{a}^{\infty} t^{-n / 2}\left(\int|V(x)| \exp \left\{-\frac{|x-y|^{2}}{2\left(1+t^{2}\right)}\right\} d x\right)^{1 / 2} d t<\infty ;
$$

then the wave operators

$$
W_{ \pm} \Psi=\lim _{t \rightarrow \pm \infty} e^{i t H} e^{-i t H} 0 \Psi, \quad \Psi \in L^{2}\left(E^{n}\right),
$$

exist.

THEOREM 4. In addition to (7) assume that there is a p satisfying $0<$ $p \leqslant 2$ such that

$$
\begin{aligned}
\left(|V|^{p} u, u\right) \leqslant C\left(\|\Delta u\|^{2}+\|u\|^{2}\right), & u \in C_{0}^{\infty}, \\
\left(|V|^{2-p} u, u\right) \leqslant C\left(\|\nabla u\|^{2}+\|u\|^{2}\right), & u \in C_{0}^{\infty},
\end{aligned}
$$

and for each $y \in E^{n}$ there is an $a>0$ such that

$$
\int_{a}^{\infty} t^{-n / 2}\left(\int|V(x)|^{p} \exp \left\{-\frac{|x-y|^{2}}{2\left(1+t^{2}\right)}\right\} d x\right)^{1 / 2} d t<\infty .
$$

Then the wave operators (10) exist.

COROLlary 5. If (7), (11), and (12) hold and $(1+|x|)^{\alpha} V(x) \in L^{p}\left(E^{n}\right)$ for some $\alpha>(2-n) / p, 0<p \leqslant 2$, then the wave operators (10) exist.

The Dirac operator is given by 


$$
L_{0}=\sum_{j=1}^{3} \alpha_{j} D_{j}+m \beta
$$

in $E^{3}$, where $D_{j}=\partial / i \partial x_{j}$ and the $\alpha_{j}, \beta$ are $4 \times 4$ matrices satisfying certain commutation relations (cf. [10]). If $Q(x)$ is a $4 \times 4$ Hermitian matrix function satisfying

$$
\sup _{x} \int_{|x-y|<\delta}|Q(y)||x-y|^{-2} d y \rightarrow 0 \quad \text { as } \delta \rightarrow 0
$$

then it was shown in [8] that $L_{0}+Q$ has a unique selfadjoint extension $L$ such that $D(L) \subset D\left(|Q|^{1 / 2}\right)$. Put $l=1$ if $m \neq 0$ and $l=0$ if $m=0$.

THEOREM 6. If $(1+|x|)^{\alpha} Q(x) \in L^{1}$ for some $\alpha>-l$, then the wave operators

$$
W_{ \pm} u=\lim _{t \rightarrow \pm \infty} e^{i t L} e^{-i t L} u
$$

exists on $\left[L^{2}\left(E^{3}\right)\right]^{4}$.

THEOREM 7. Assume there is a $p$ satisfying $0<p \leqslant 2$ and an $\alpha>-l / p$ such that $(1+|x|)^{\alpha} Q(x) \in L^{p}$. If $q=\max (p, 2-p)$ and

$$
\sup _{x} \int_{|x-y|<\delta}|Q(y)|^{9}|x-y|^{-2} d y \rightarrow 0 \quad \text { as } \delta \rightarrow 0
$$

then the wave operators (15) exist.

\section{REFERENCES}

1. J. M. Cook, Convergence of the Moller wave-matrix, J. Mathematical Phys. 36 (1957), 82-87. MR 19, 1011.

2. J. M. Jauch, Theory of the scattering operator, Helv. Phys. Acta 31 (1958), 127158. MR 20 \# 682 .

3. S. T. Kuroda, On the existence and unitary property of the scattering operator, Nuovo Cimento 12 (1959), 431-454.

4. Tosio Kato, Perturbation theory for linear operators, Springer-Verlag, Berlin and New York, 1966. MR 34 \# 3324.

5. R. T. Prosser, Relativistic potential scattering, J. Mathematical Phys. 4 (1963), 10481054. MR 27 \# 4509.

6. K.-J. Eckardt, On the existence of wave operators for Dirac operators, Manuscripta Math. 11 (1974), 359-371. MR 49 \# 3346.

7. Martin Schechter, Scattering theory for elliptic operators of arbitrary order, Comment. Math. Helv. 49 (1974), 84-113. MR 51 \# 3726.

8. - Nonhomogeneous elliptic systems and scattering, Tôhoku Math. J. 27 (1975), 601-616.

9. - Spectra of partial differential operators, North-Holland, Amsterdam; American Elsevier, New York, 1971.

10. L. I. Schiff, Quantum mechanics, McGraw-Hill, New York, 1968.

11. Krešimir Veselić and Joachim Weidmann, Asymptotic estimates of wave functions and the existence of wave operators, J. Functional Analysis 17 (1974), 61-77. MR 49 \#11067.

BELFER GRADUATE SCHOOL OF SCIENCE, YESHIVA UNIVERSITY, NEW YORK, NEW YORK 10033 